\title{
Grounded design of web-enhanced case-based activity
}

\author{
Hyeonjin Kim • Michael J. Hannafin
}

Published online: 17 October 2006

(C) Association for Educational Communications and Technology 2006

\begin{abstract}
Cases have been used in education through a variety of methods, such as case written analysis, case discussion, and case development. Recent case definitions and uses have extended their traditional uses. In case-based reasoning, cases are considered to represent knowledge, and the use of cases is integral to an individual's problem-solving process. Case-based activity (CBA) builds on CBR research and theory, emphasizing the transition of novices toward experts' reasoning and applications. The purpose of this paper is to present the theoretical assumptions and principles for designing grounded, web-enhanced CBA. Findings from recent implementations and implications for design are presented.
\end{abstract}

Keywords Case-based reasoning - Grounded design ·

Technology-enhanced learning environments

\section{Introduction}

Cases have been used in education for many years through a variety of methods (e.g., case written analysis, case discussion, and case development). As an instructional method, cases may bridge theory and practice by allowing

H. Kim (ه)

Policy Planning Office, Korea Education \& Research Information Service,

22-1 KERIS Building, Ssangnim-dong,

Jung-gu, Seoul 100-400, Korea

e-mail: jinnie@keris.or.kr

M. J. Hannafin

Learning \& Performance Support Laboratory,

The University of Georgia, 611 Aderhold Hall,

Athens, GA 30602, USA

e-mail: hannafin@uga.edu 
students to engage in authentic, contextualized learning experiences (Masoner, 1988; Shulman, 1992). Typically, case methods present complex contexts in the format of dilemmas or problem-solving narratives.

Recently, theorists have characterized "cases" as individual knowledge represented and retrieved in the form of stories (Brown, Collins, \& Duguid, 1989; Kolodner, 1993; Lave \& Wenger, 1991; Schank, 1999). From this perspective, the use of cases is not limited to instruction (i.e., case methods), but also represents human habits of thinking. Recent work based in case-based reasoning (CBR) theory (e.g., Kolodner, Owensby, \& Guzdial, 2004; Schank, Berman, \& Macpherson, 1999) has potential implications for designing alternative approaches to, and explaining the effects of, case-based learning. The purpose of this paper is to present a framework for designing one such extension, web-enhanced case-based activity (CBA), which is consistent with and extends CBR's foundations and assumption.

\section{A CBR primer}

Experts reference their experiences when problem solving, retrieving memories deemed relevant to given problems or situations (Ericsson \& Lehmann, 1996; Ericsson \& Smith, 1991). For example, chess experts retrieve sophisticated memories regarding the characteristics of chess positions, appropriate playing methods, and alternative moves (see de Groot, 1978). Experts across domains share several performance characteristics (Anderson \& Leinhardt, 2002; Anzai, 1991; Ericsson \& Kintsch, 1995; Feltovich, Johnson, Moller, \& Swanson, 1984): They possess a rich body of available knowledge which they readily access and apply during problem solving. Experts refine knowledge structures (i.e., schema) evolve through experience and help them to assess new situations instantaneously, separate important from unimportant detail, and quickly identify problems and develop solutions. In contrast, the novices' knowledge is neither rich nor readily accessible. They often expend significant time and effort in interpreting situations, tend to focus on irrelevant information, and fail to adequately identify problems or develop solutions. Researchers report that while experiences per se do not always promote expertise, extended, deliberate, purposeful practice tends to improve performance and refine expertise (Ericsson, Krampe, \& Tesch-Römer, 1993; Ericsson \& Lehmann, 1996).

CBR provides a useful theory for examining how deep understanding and expertise emerge through experiences. As illustrated in Fig. 1, the CBR theory posits how individuals (including experts and novices) think, reason, and act based on experiences in the form of cases (Kolodner, 1993; Kolodner et al., 2004; Riesbeck, 1996; Schank, 1999; Schank et al., 1999). According to the CBR model, when encountering a problem, we retrieve-often unconsciously-previous experiences in the form of cases considered relevant to a new situation. Personal case libraries are indexed, which serves to structure individual case knowledge. 


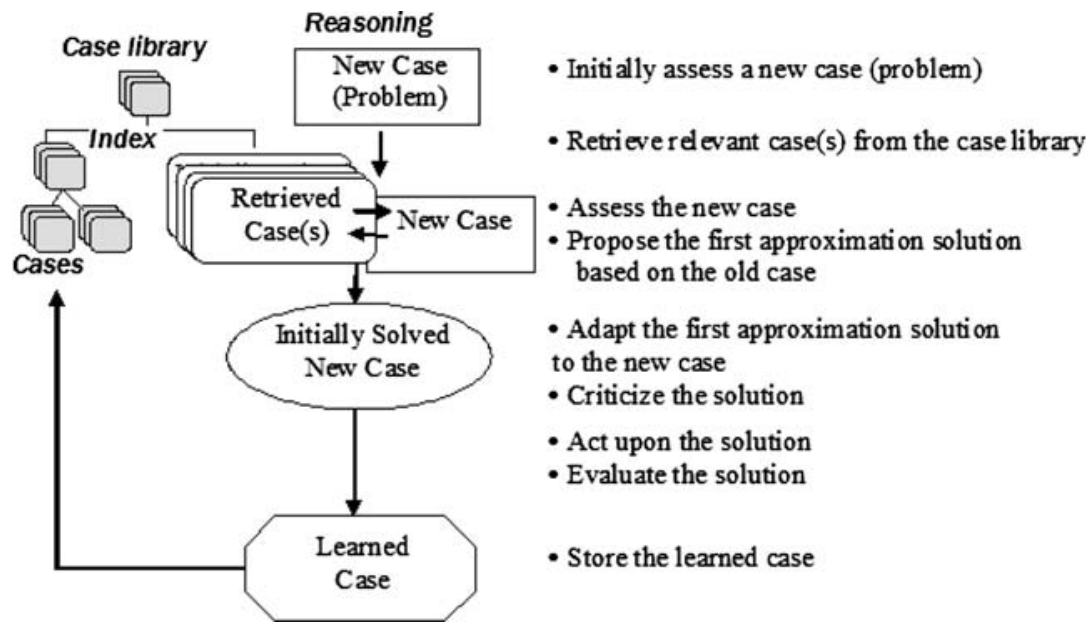

Fig. 1 Case-based reasoning (CBR) process Note. Adapted from Aamodt and Plaza (1994), Kolodner (1993)

Indexing schemes can also codify the critical domain concepts and problem-solving strategies of a community. Lacking background knowledge and experience, novices' case libraries are limited and provide little indexing; experts, in contrast typically have highly indexed and extensive case libraries, reflecting rich and varied experience in particular domains (Kolodner \& Guzdial, 2000; Schank et al., 1999).

After retrieving a relevant previous case, we generate a first approximation solution based upon its contents, our interpretation of the new case, and alternative solutions. Resulting actions are then evaluated during and after application to real-world problems. When unable to solve a new problem based on previous case knowledge, we encounter "expectation failure" (Schank et al., 1999, p. 171). An iterative process ensues, with candidate solutions evaluated and applied until the requirements of the new case are satisfied. Through repeated experience, we develop richly situated domain knowledge and experiences, enabling sophisticated organizing, indexing, and applying of our case knowledge.

For educational applications, CBR provides a key grounding theory for designing learning environments. Novices gain and refine their expertise by scaffolded interactions with expert case knowledge. Since they lack their own cases knowledge (i.e., experiences), novices may access and use the exemplary cases to address their problems or accomplish their tasks. CBR-based learning environments, in effect, provide apprentice-like experiences where novice activities are scaffolded using experts' video- or text-based case knowledge. In this paper, we examine how CBR design principles are instantiated using web technology to make expert case knowledge accessible to novices and describe a framework for grounding design practices. 


\section{Grounded design of web-enhanced CBA}

According to Hannafin, Hannafin, Land, and Oliver (1997), grounded design involves "the systematic implementation of processes and procedures that are rooted in established theory and research in human learning" (p. 102). Grounded design allows comprehensive interpretation, extrapolation, and application of research and theory for designing learning environments. Figure 2 illustrates Kim, Hannafin, and Thomas (2004) CBA design framework. CBA provides concrete tasks and activities (scenario work, what's the story, planning, doing, and telling your story), instructor support, peer review, and web resources. Table 1 summarizes how the foundations and assumptions of grounded design are applied in CBA design. While each is isolated for clarity, the design foundations are interdependent in practice.

\section{Situated knowledge}

Psychological foundations emphasize how we think and learn, guiding both the specification of learning goals and associated pedagogical approaches. Consistent with the perspectives of CBR proponents (i.e., Brown et al., 1989; Kolodner et al., 2004; Lave \& Wenger, 1991; Schank et al., 1999; Stevens, Collins, \& Goldin, 1982), CBA learning environments are situated and constructivist-inspired, knowledge is assumed to evolve through repeated experiences and ongoing support.

Accordingly, two CBR assumptions are critical in guiding both the identification of learning goals and pedagogical approaches: development of case libraries and participation in the social practices of a community (Kolodner et al., 2004; Schank, 1999). The development of case libraries through CBR experiences is consistent with assumptions as to how situated knowledge is refined through repeated experience. Case libraries comprise cases and indexes that are situated in particular experiences. An individual case is represented as knowledge in the form of stories (Lave \& Wenger, 1991),

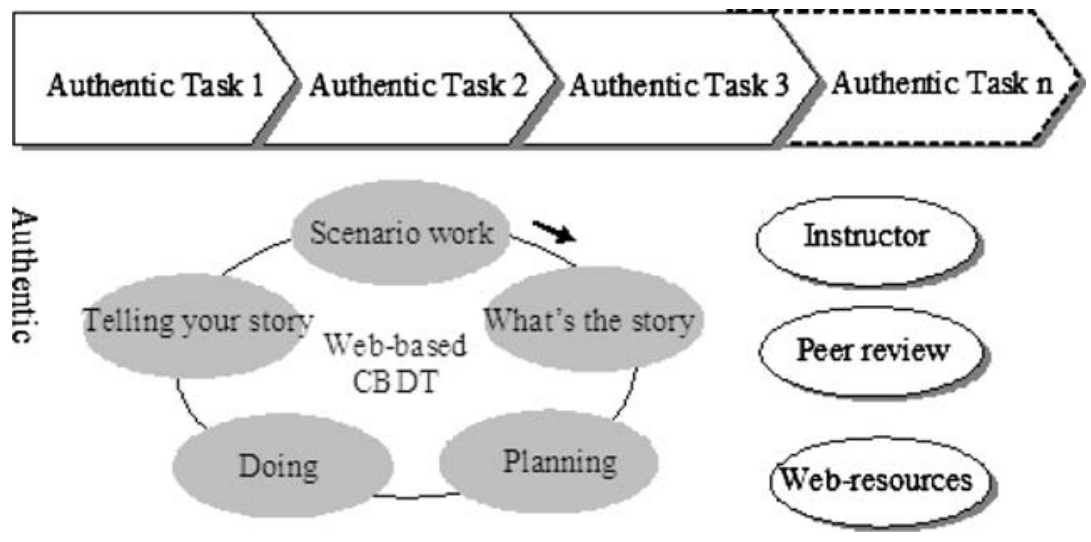

Fig. 2 The structure of case-based activity (CBA) 


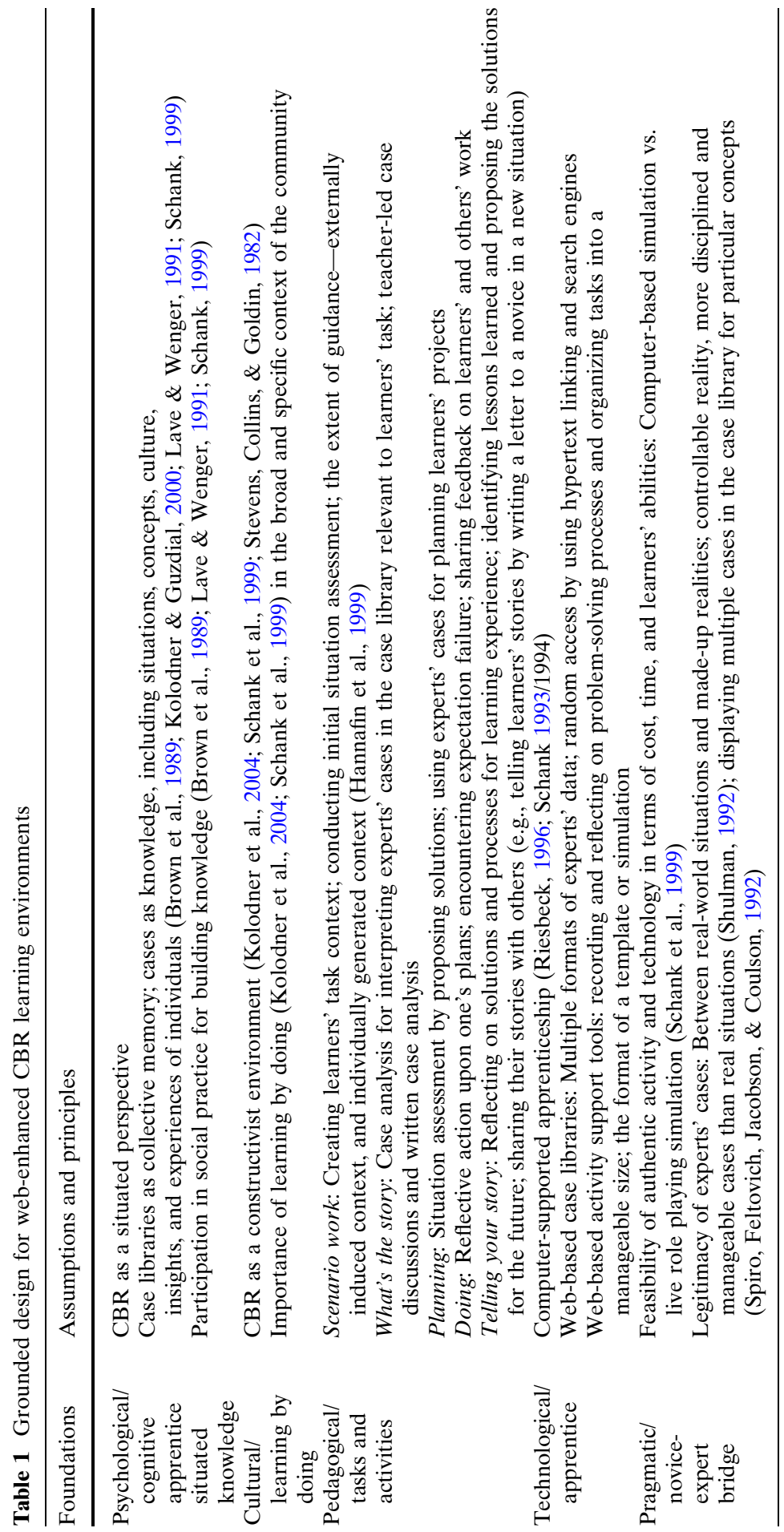


which reflect the cultural values, insights, and experiences of individuals (Brown et al., 1989; Shulman, 1992). The individual case is indexed in terms of purposes, as well as similarities and differences among cases. Expert case indexing represents the major concepts of the domain and their relationships as understood by individuals with extensive experience or expertise (Kolodner \& Guzdial, 2000). For example, expert teachers recognize the relationship between and among students, content, and pedagogy, identifying ways in which student characteristics jointly influence the selection of content and pedagogy. Thus, the teaching community has identified critical attributes of and relationships among elements of expertise, including knowledge of students, curriculum, content, and pedagogy (Leinhardt \& Greeno, 1986; Shulman, 1986).

Case knowledge becomes situated in the social and physical contexts of a community as it is repeatedly applied in new contexts (Brown et al., 1989; Schank, 1999); therefore, participation in social practices helps to build the knowledge valued by and relevant to a given community. Novices become increasingly knowledgeable as they communicate with other members (particularly seasoned members), use their knowledge, and otherwise experience the practices of a community. Although CBR's situated perspective focuses on the individual nature of learning and cognition, case knowledge and experiences are shaped by the social practices of a community (Schank, 1999). Consistent with cognitive apprenticeship models, therefore, web-based CBR learning underscores the importance of experts' guidance for scaffolded experiences (Schank, 1994/1994; Stevens et al., 1982).

\section{Learning by doing}

Typically, CBR-based learning environments emphasize learning-by-doing as a way to both experience and learn, increasing the authenticity and meaningfulness of the experience. Learning-by-doing activities and values are aligned with those embraced by a given culture or community. For example, teacher educators and researchers suggest that prospective teachers will both learn about and implement technology more successfully in their future classrooms when preservice experiences are situated in authentic teaching problems and experience (Office of Technology Assessment, OTA, 1995; Russell, Bebell, O'Dwyer, \& O'Connor, 2003). Lacking opportunities for firsthand experience integrating technology into their own classrooms, beginning teachers appear to benefit more from examining expert teachers' perspectives and experiences in authentic classroom contexts than from focusing on technology mechanics (Ertmer et al., 2003). Kim et al. (2004), for example, implemented a web-enhanced, CBR course for preservice teachers consistent with the values of teacher education and technology integration communities by providing authentic teaching problems and activities (e.g., integrating software into instruction, developing concept maps for lesson presentation, implementing technology-enhanced lesson activities). Learning-by-doing reflected both the overarching community value and established the 
associated design requirements for individuals preparing to enter the technology integration teaching community.

Tasks and activities

According to Hannafin et al (1997), pedagogical foundations refer to how content is represented and affordances provided to support learning in accordance with specific foundation theories. The pedagogical foundations of CBR support situated learning by enabling novices to engage in authentic tasks as an apprentice under the tutelage of virtual and live experts. As previously illustrated in Fig. 2, CBA consists of scenario work, what's the story, planning, doing, and telling your story for the completion of authentic tasks.

Scenario work is designed for novices to develop or select their task contexts by identifying relevant contextual information, goals, deliverables, and scope. Novices develop or select their task scenario based on their experiences or available scenario examples. Graduate students in an instructional design course, for example, determine task contexts, including the target audience, content area, and educational needs that correspond to their interest or needs. The contexts influence subsequent decisions, such as media, instructional methods, and level of content.

Scenario work supports novices in initial situation assessment. In principle, once novices determine personally relevant task scenarios, they develop expectations for their tasks (Schank et al., 1999) by assessing why they chose the scenario, whether completion is feasible, and which resources are needed. In practice, since novices typically lack a deep understanding of the task context and important issues, they tend to develop superficial scenarios. Their scenarios are often too simple to be worthwhile or too complicated or unspecified to be resolved (Kim, 2005). Therefore, novices require scaffolding to ensure that their scenarios include critical concepts of the given community and manageable complexity. For example, problem-based learning (PBL) in medical schools (Barrow, 1985) often provides students with an actual patient case, including important concepts of basic science and clinical processes. The case generally consists of a description of the presenting problem and how it was diagnosed and treated. Typically, students are asked to formulate a hypothesis on the patient's problem based on an apparent symptom by identifying critical concepts and relationships between data and diagnosis. Like PBL, externally induced contexts serve to bridge the novice-expert gap by framing a situation that learners must interpret, identify and generate specific problems to be addressed (Hannafin, Land, \& Oliver, 1999). The scenarios learners generate individually, in contrast, become increasingly useful as learners mature, become knowledgeable, and gain additional experience.

What's the story is designed to support interpretations and analyses of experts' exemplar cases relevant to the learner's tasks and scenarios. Whereas experts readily utilize the knowledge and skills represented in their case libraries, novices do not; rather, they tend to oversimplify or poorly interpret case libraries based on their limited understanding 
(Schank, 1999; Spiro et al., 1992). Left unassisted, novices typically engage in trial and error activities (or are unable to interpret tasks or events). By deliberately examining expert-generated cases relevant to their needs, novices engage in tasks and events otherwise beyond their individual capabilities (Kolodner et al., 2004; Schank, 1999).

Opportunities for case interpretation are provided during case discussion and written case analysis after learners watch or read experts' cases that are similar to their task cases. Case discussions are often led by a facilitator who leads question and answer sessions that guide novices to concepts otherwise overlooked. Moreover, facilitated case discussion stimulates dialog in the form of stories between old and new members and extends community lore, experiences, and practices (Carter, 1989; Lave \& Wenger, 1991). For written case analysis, guidance is needed to support understanding of experts' cases; unguided, novices tend to analyze experts' cases based only on their limited understanding and subjectivity. Therefore, templates (e.g., prompt questions or titles) should provide critical concepts and components for understanding experts' knowledge and practices so that novices can focus on important issues in the cases.

For example, the Case Interpretation Tool in Owensby and Kolodner's (2002) Case Application Suite enables learners to analyze and understand expert cases relevant to their design work. Learners describe their case analysis using prompts, such as time and location, problems, benefits, solution(s) chosen, alternative solutions, solution implementation, technology used, criteria applied, and advice for others. Detailed prompts are provided, such as: "Where and when did this challenge take place? Be as specific as possible in telling the sequence of events." Hints are also provided to support case analysis (e.g., "Think about that place and time and the effect that it had on the challenge the experts faced"') (Kolodner et al., 2004, p. 840). Their Case Interpretation Tool subsequently helps novices to develop their own cases by guiding their initial interpretation and understanding of expert cases. Since cases are analogs and not identical to the unique situations novices encounter, interpretation helps to clarify experts' experiences and relate them to the novice's own cases (Riesbeck, 1996).

During planning, novices brainstorm ideas and plans for completing their task scenarios. They conduct situation assessments for their new tasks, identify possible solutions for their goals, choose first approximation solutions among candidate ideas, and anticipate the results and pitfalls of the solutions. In the planning phase, expert cases scaffold the process of applying the expert's ideas to the novice's scenario, attempting to highlight how experts address comparable dilemmas. This can prove difficult for novices, as they are often unable to distinguish which cases are applicable and what concepts are useful-that is, the similarities and differences between an expert's cases and their own situations (Kolodner et al., 2004). Deliberate scaffolding helps novices as they are given the information about relevant cases and the prompts of critical concepts. For example, the Case-Application Tool helps learners to determine whether their case interpretations are applicable to their 
projects by prompting to consider goals, issues, criteria and constraints, such as "describe the action, design, or choice you are working with" (Kolodner et al., 2004, p. 853).

Next, the doing phase is designed to support novices as they act on their plans (Kolodner et al., 2004; Schank et al., 1999). Doing, for example, may include developing products, playing golf, or operating an automobile. Although doing focuses on performing, it also requires that individuals reflect on their actions by comparing and revising their plans. Learners encounter expectation failures when their plans do not work as intended, providing opportunities to evaluate plans and to conduct deeper situation assessments. Expectation failure, therefore, provides teachable moments for supporting or disproving working theories and assumptions. To scaffold doing, guidance such as prompts and feedback can be provided. For example, in Schank et al.'s (1999) Advise the President, learners are guided in both planning and doing through The Scenario Operations. Learners use Scenario Operations to provide direction for their decisions and activities via guidance such as, "asking experts for opinions on topics relevant to completing the report, compiling information for future reference, making claims about strategies, and backing up claims with selections from the information the students compiled" (pp. 175-176).

Peer collaboration is also important in the doing phase. As individuals generate and share ideas, they practice how to articulate ideas and warrant claims while encountering alternative, sometime competing, perspectives (Brown et al., 1989). These interactions introduce novices to the culture and conventions of a given community of practice as they learn to share, interact with, and critique the ideas and actions of peers (Brown et al., 1989; Lave \& Wenger, 1991).

Since CBR assumes that humans store and use knowledge in the form of stories, the telling your story prompt is designed to support transfer as individuals reflect holistically on their experiences. Learners construct and share their stories, formalizing and indexing their case knowledge. Participants briefly describe their experiences in the form of stories using both specific concrete, contextual incidents and their perceptions about the events. It is important that participants reflect on critical incidents and concepts related to their personal accounts, as they identify lessons learned, analyze reasons for impasses, and propose alternatives to address future problems or dilemmas. Individual lessons learned may include a broad range of knowledge and skills, such as conceptual understanding, beliefs, and know-how.

After learners reflect on their activities, it is important to share their stories by developing cases for peers in subsequent semesters (e.g., Stable cited in Kolodner et al., 2004), posting cases and participating in follow-up discussions, and simulating advice for using their cases. Because social practice through conversations and collaboration are central to learning communities (Brown et al., 1989; Lave \& Wenger, 1991), activities for sharing stories may increase reflection and transfer of learning. For example, Help Susan is designed to help 
learners write a letter to a peer novice who may encounter a similar dilemma or challenge in a different situation, allowing the learners to examine potential transfer to new situations (Kim, 2005). Letters may also promote membership in the community by relating the experiences of a slightly more seasoned practitioner to an inexperienced novice (Lave \& Wenger, 1991).

\section{Computer-facilitated apprenticeship}

In grounded design practice, technological foundations refer to how media are available to support learning (Hannafin et al., 1997), such as to simulate real-world tasks and to conduct meetings with virtual advisors. For example, several researchers have implemented learning-by-doing approaches that enable novices to engage in case-based problems or tasks using the affordances of computer technology (see, for example, Kolodner, Hmelo, \& Narayanan, 1996; Kolodner et al., 2004; Schank et al., 1999).

Web affordances support a range of CBA, including the creation of and access to expert and individual case libraries and learning activities. Figure 3 presents an example of the case-based doing tool (CBDT) designed to help preservice teachers develop technology-enhanced lesson plans and instructional materials (Kim et al., 2004). CBDT presents expert teachers' case libraries and a support tool for preservice teachers who typically lack the experience, as well as a real classroom context, to engage in real-life applications of technology in everyday teaching.

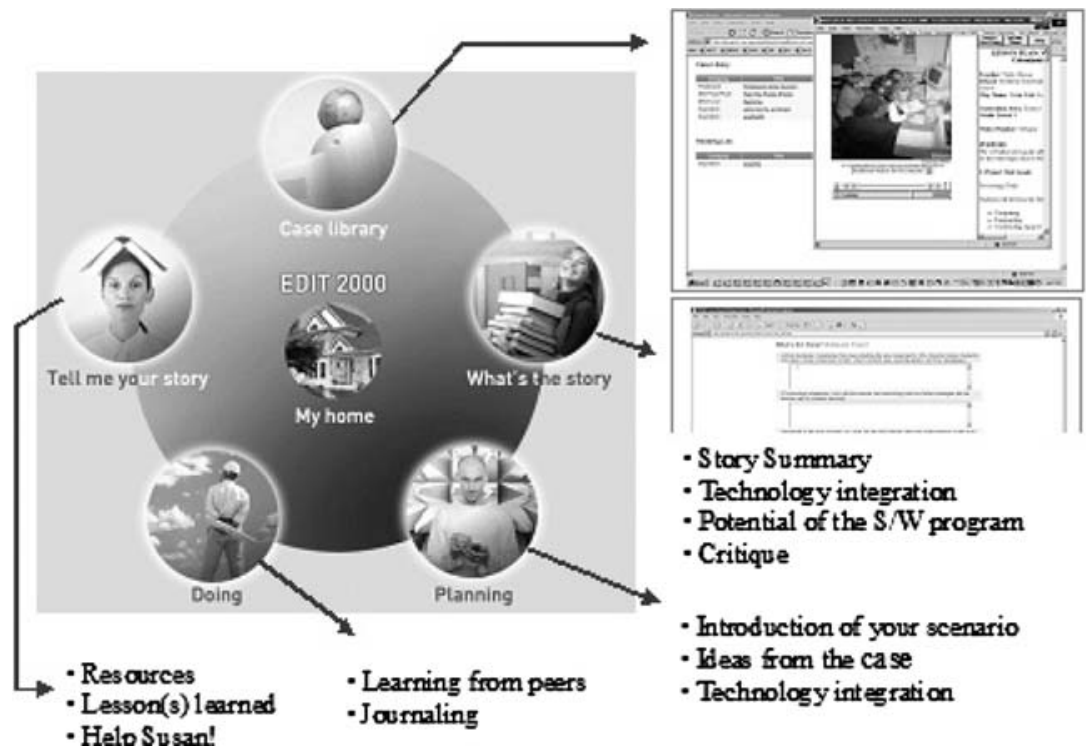

Fig. 3 The screen shot and template structure of the web-based CBDT (Adapted from Kim, Hannafin, \& Thomas, 2004) 


\section{Web-based case library}

Case libraries are assembled by collecting and indexing expert (and peer) exemplary cases. In CBDT, cases reflect "the presence of experts" (Riesbeck, 1996, p. 59), including their situated knowledge and perspectives that function to advise or guide. In CBDT, cases include experts' stories (via interviews) in the form of narratives that enable novices to better understand the expert's interpretation, reasoning and action in context.

While the expert instructor can draw upon a wealth of stories or demonstrations based on personal experience, novice teachers often cannot relate to them due to limited real-world teaching experience (Carter, 1990). Multimedia cases provide multiple ways to present cases and relevant contextual information to increase both authenticity and meaningfulness. Web affordances, such as hypertext links and search engines, allow rapid access to expert cases and independent resources. In CBA, novices access experts' interviews, problem and performance context, work samples, and archival data for each case (Kim, Hannafin, \& Kim, 2005). Because tools help to simulate the retrieving of memories in personal case libraries, learners can also practice finding and using information during their own problem solving. For example, in a web-based case library for a Turfgrass management course (Colaric, Turgeon, \& Jonassen, 2002/2003), cases were organized to reflect both basic information (e.g., title, geographic location, turf species at the problem location, turf type, brief description of the cases) as well as a framing and solving framework related to course tasks (e.g., actions for correcting the problem, rationale for actions, expected results, results of actions, and unexpected results). When learners select a further option, a pull-down menu consisting of short case descriptions is returned based on the initial selection. Learners choose cases and engage in the complete story via photos, instructional modules, and hypertext links.

\section{Web-based activity support tool}

Activity support tools afford opportunities for novices to apprentice under seasoned and experienced mentors via templates, simulations, and live teachers (Riesbeck, 1996; Schank, 1993/1994; Stevens et al., 1982). Novices can become overwhelmed by the complexity and ill-structured nature of authentic problems; technology, therefore, assists them in identifying and organizing problems (Kolodner et al., 2004). The tools also guide novices to focus on critical concepts and strategies of the experts' problem-solving processes. The Reflective Learner tool, for example, prompts students (1) to identify a problem during the project, (2) to describe their solution to the problem, (3) to address lessons learned from the experience, and (4) to anticipate similar situations (Turns, Newstetter, Allen, \& Mistree, 1997). Students reported that these activities helped them to understand what they were doing (and why), and enabled them to better locate reasoning processes embodied within the case. 
Bridging knowledge and experience gaps

Pragmatic foundations address unique situational constraints associated with design and implementation, ensuring practical designs that are appropriate to given problems and settings (Hannafin et al., 1997). The authenticity of CBR activities allows participants to gain experience in the essential practices of a community. Novices, however, are often unable to follow or understand initial activities that are complex; the novice's transition into a community of practice requires the scaffolding of CBA activities (Lave \& Wenger, 1991).

Scaffolding can be provided by computer-based tools, the instructor, peers, and web resources to clarify the context of the performance, support actions, and otherwise guide as novices engage in problems or tasks. For example, live goal-based scenarios can be utilized for role-playing simulations in situations where computers are not available or appropriate (Schank et al., 1999). Live scaffolding may assume the form of standard scripts or dynamic expert feedback before, during, or subsequent to performance. Learners can practice communication, human interactions, and reasoning as well as develop and refine domain knowledge and skills.

The relevance and authenticity of cases is central to CBA. The model enables novices, otherwise unable to participate in real-world experiences, to engage in authentic problems and activities while in classroom settings. Cases, a blend of actual and realistic situations, provide a bridge between theory and practice as "a piece of controllable reality, more vivid and contextual than a textbook discussion, yet more disciplined and manageable than observing or doing work in the world itself" (Shulman, 1992, p. xiv). To this end, cases enable opportunities for problem solving and simulated decision-making where actual experience is impractical, too time-consuming, or exceedingly complex for novices to otherwise engage in (Spiro et al., 1992).

\section{Implications for design}

Web-enhanced CBA has been developed conceptually and studied via repeated implementations (Kim, 2005; Kim \& Hannafin, 2005a, 2005b). As shown in Table 2, several implications for design based are evident.

\section{Designing and indexing experts' case libraries}

Case libraries are designed to capture experts' stories-their experiences, knowledge, strategies, beliefs, and insights. Novices can draw on experts' wisdom, as they might do while apprenticing under live experts. Integrating New Technologies Into the Methods of Education (InTime) (Krueger, Boboc, Smaldino, Cornish, \& Callahan, 2004), for example, provides online video vignettes for 60 lessons featuring PreK-12 teachers integrating technology into their classrooms. Web-based video cases capture real classroom situations, expert teachers' stories, and artifacts of the teachers' lesson plans. Video 


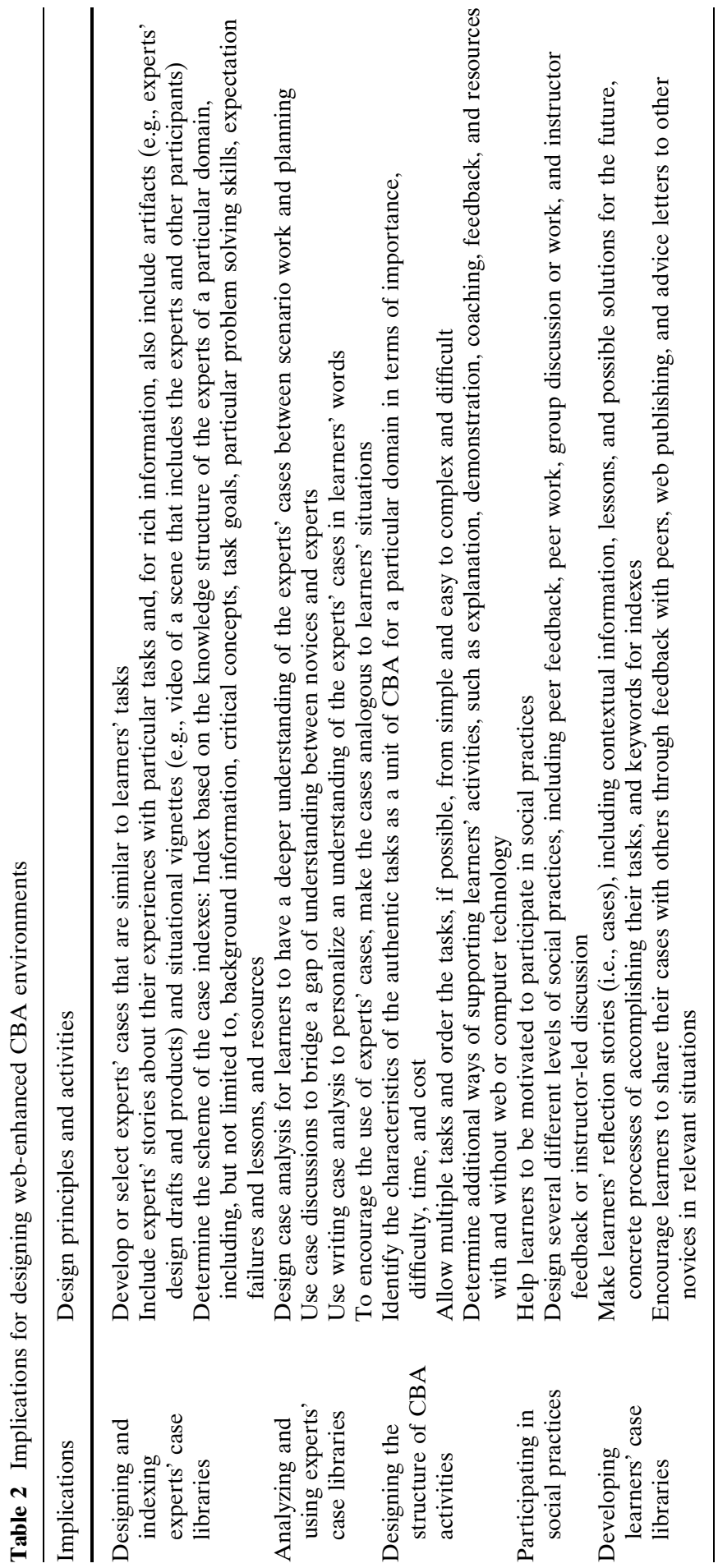


clips can also be searched based on software program demonstrated, grade level taught, subject area, learning elements, and other options. After implementing InTime for three semesters, more than $40 \%$ of the 31 university faculty members reported that preservice teachers' standards-based knowledge and skills for teaching with technology improved in all areas (e.g., Technology Operations and Concepts, Planning and Designing TechnologyEnhanced Learning Environments and Experiences) as compared to previous preservice teachers.

Accordingly, designers or instructors must select and index experts' cases that represent the specific practices emphasized. Case indexing is especially important because it serves to formalize experts' knowledge structures, such as background information (e.g., when, where, who), critical concepts, task goals, particular problem solving skills (e.g., time management), expectation failures, lessons, and resources (Kolodner et al., 2004; Schank et al., 1999).

\section{Analyzing and using experts' case libraries}

Expert analysis helps novices to vicariously understand expert knowledge, problem-solving strategies, and reasoning. Case discussions and writing, therefore, need to amplify the experts' understanding of the specific knowledge, skills, and perspectives of the corresponding community (Kolodner et al., 2004; Schank et al., 1999). Further, using the results of the original case analyses helps novices to deepen and formalize their understanding of experts' case knowledge by summarizing key ideas that emerge during analyses and articulating relevance for their own purposes. In a series of studies, preservice teachers reported that case analysis both expanded and deepened their understandings of teaching with technology by focusing on strong and weak points, possible obstacles, and the extent of technology's usefulness (Kim, 2005; Kim \& Hannafin, 2005b). However, the preservice teachers did not simply adopt practices when they perceived the experts' cases as differing from their own; rather, they focused on comparing the situations rather than the specific strategies or solutions. Case substance and context, therefore, are important to both design and use of expert cases. When sufficiently analogous to situations encountered by novices, cases both embody the reasoning of the expert and guide novices in their reasoning (Kolodner et al., 2004).

\section{Designing CBA structures}

CBA should enable novices to engage in experts' authentic experiences and activities in "safe" settings. CBA needs to encourage hands-on or "doing" activities involving authentic tasks and realistic settings, which represents the contexts, activities, resources, and reasoning of a corresponding community (Kolodner et al., 2004; Schank et al., 1999). Activities must also approximate reasonably the situational complexity, cost, and time demands of a case. Therefore, designers or instructors must first identify the characteristics of the authentic tasks for a particular domain in terms of importance, difficulty, time, 
and cost. For example, in the web-enhanced CBA course for learning to teach with technology (Kim et al., 2004), ordinary practices of professional educators that preservice teachers need to follow were identified as the development and implementation of technology-enhanced lesson plans and materials. Course projects were structured and sequenced in terms of importance and difficulty, beginning with a simple graphing program and culminating with WebQuest lesson planning and material development.

This design structure, therefore, scaffolded novices' learning, thereby coordinating the complexity of authentic tasks (Kim, 2005). That is, initially, preservice teachers retained their beliefs about technology use in education and used computer technology mainly as an add-on activity. However, the final task-planning and developing a student-centered constructivist learning WebQuest lesson-required greater task complexity, content integration, and technology integration than previous projects. During this phase, all five preservice teachers extended their understanding of the potential of technology and connected its role to student thinking and learning. The preservice teachers' reports and interviews demonstrated that they began to recognize and share the values and practices of the teaching with technology community. CBA structures for novices, therefore, need to be sequenced to support the novice-expert transition. In addition, designers and instructors need to identify other alternatives to strengthening participant activities, such as providing instructor-expert explanations, demonstrating, providing feedback, coaching, and guiding to other resources.

\section{Participating in social practices}

CBR provides an important way to learn from and participate in the practices and perspectives of a community. Instructors, as experts, scaffold by providing explanations, demonstrations, feedback, formative progress assessments, and alternative approaches when novices encounter impasses. Instructors can also relate personal experiences beyond those documented in expert case libraries. In two research studies (Kim, 2005; Kim \& Hannafin, 2005b), preservice teachers viewed the instructor as a model and coach who demonstrated and advised on technological skills, pedagogical ideas, and specific lesson-planning skills. During two semesters, the instructor recounted conversations about her own teaching practices, and preservice teachers' conversations increasingly indicated the characteristics of exemplary teachers' social practices for teaching with technology that researchers have identified in literature (e.g., subject-specific conversations, Windschitl \& Sahl, 2002). The preservice teachers, for example, asked about the appropriateness of the topics and learning activities in developing their lessons. The instructor also reported that she helped preservice teachers organize their ideas for and expand on their lessons, stating: "[that was the] kind of thing that I didn't get to do in the other classes."

Peer collaboration, such as peer feedback and collaborative work, also provides important opportunities to participate in social practices (Lave \& 
Wenger, 1991). In previous CBA implementations (Kim \& Hannafin, 2005a), preservice teachers identified peer review as particularly helpful. Based on both reports documented via the CBA doing prompt as well as interviews, preservice teachers' conversations focused extensively on practices for teaching with technology and not strictly technical issues. When topics of conversation are situated in authentic tasks and activities, conversations share the lore, skills, beliefs, and culture of the community (Lave \& Wenger, 1991). Peer review also allowed preservice teachers to engage in the social practices of professional teachers and to share their work.

Developing learners' case libraries

The role of individual case libraries is to facilitate meaningful reflection on and transfer of knowledge and skills (Kolodner et al., 2004; Schank et al., 1999). Novices are encouraged to document their own indexed stories, including key concepts and insights during task completion-a natural way of storing (Schank, 1999) and sharing knowledge and experience with members of a community (Lave \& Wenger, 1991).

Reflections include the processes used while accomplishing a task, evaluating solutions, developing alternatives, considering difficulties and task complexity, managing time, and contemplating future applications. During the reflection phase of previous implementations (Kim, 2005), preservice teachers were asked to describe the resources used, lessons learned, and the focus of their letter to a novice colleague (i.e., telling your story). All identified lessons learned from their CBA projects, such as ways to improve products (e.g., reasonable lesson activities for limited time) and the process for completing projects (time allocation and timely decision making).

Case libraries may also be refined by sharing feedback among peers, web publishing, and advising other novices (Kim, 2005; Kolodner et al., 2004). In the two research studies (Kim, 2005), writing a letter (i.e., Help Susan!) enabled the preservice teachers to report their experiences to a colleague in a related but new situation, allowing them to practice possible transfer of their learning. CBD reports described how they built and used their case library to solve new situations using technology with their teaching. For example, preservice teachers documented insights associated with applying or adapting their approach, such as insufficient time for a WebQuest lesson, reducing the number of tasks, infusing content, and simplifying student activities.

\section{Conclusion}

By applying grounded design principles, CBA provides an important approach-especially for situated learning with cases. Although there are few longitudinal studies on how novices become experts through CBA or CBR approaches, several projects and research studies report transfer of learning and enculturation in the corresponding community (Kim, 2005; Kim \& Hannafin, 
2005a, 2005b; Kolodner et al., 2004; Schank et al., 1999). Researchers also report that through CBR activities, novices gained opportunities to assess experts' actual situations as well as their own project contexts (Kim, 2005; Kolodner et al., 2004). Situation assessment helps to cultivate reflective thinking (Schön, 1983), an important characteristic of expert performers. Novices may initiate, then refine, their thinking habits through multiple, progressively sophisticated CBA experiences.

Through technology-enhanced CBA, novices may gain access to the experience and wisdom of veteran members of a community, helping them to understand the experts' culture of practice and decision-making, thus improving their reasoning skills. However, while case libraries have a rich history in computer science (Kolodner \& Guzdial, 2000), the technologies and methodologies of teaching and learning are still emerging. Grounded design principles should help to guide emergent technology-enhanced approaches, ensuring that both design processes and pedagogical activities are informed by, and contribute to, available research, theory, and practice.

The effectiveness of CBA learning environments needs to be verified through iterative implementation. Many researchers have emphasized the importance of longitudinal study for newly designed learning environments in order to deeply understand complex educational phenomena (Design-Based Research Collective, 2003; Reeves, Herrington, \& Oliver, 2005; Wang \& Hannafin, 2005). Through iterative implementation, designers may better align the learning environments with CBR theory.

\section{References}

Aamodt, A., \& Plaza, E. (1994). Case-based reasoning: Foundational issues, methodological variations, and system approaches. AI Communications, 7(1), 39-52.

Anderson, K. C., \& Leinhardt, G. (2002). Maps as representations: Expert novice comparison of projection understanding. Cognition and Instruction, 20(3), 283-321.

Anzai, Y. (1991). Learning and use of representations for physics expertise. In K. A. Ericsson, \& J. Smith (Eds.), Toward a general theory of expertise: Prospects and limits (pp. 64-92). Cambridge; New York: Cambridge University Press.

Barrow, H. S. (1985). How to design a problem-based curriculum for the preclinical years. New York: Springer Pub. Co.

Brown, J. S., Collins, A. M., \& Duguid, P. (1989). Situated cognition and the culture of learning. Educational Researcher, 18(1), 32-42.

Carter, K. (1989). Using cases to frame mentor-novice conversations about teaching. Theory Into Practice, 27(3), 214-222.

Carter, K. (1990). Meaning and metaphor: Case knowledge in teaching. Theory Into Practice, 29(2), 109-115.

Colaric, S. M., Turgeon, A. J., \& Jonassen, D. H. (2002/2003). Development of a web-based case library. Journal of Educational Technology Systems, 31(1), 63-70.

Collins, A. M., Brown, J. S., \& Newman, S. E. (1989). Cognitive apprenticeship: Teaching the craft of reading, writing and mathematics. In L. B. Resnick (Ed.), Knowing, learning, and instruction: Essays in honor of Robert Glaser (pp. 453-494). Hillsdale, NJ: Erlbaum.

de Groot, A. D. (1978). Thought and choice in chess. Mouton: The Hague.

Design-Based Research Collective (2003). Design-based research: An emerging paradigm for educational inquiry. Educational Researcher, 32(1), 5-8. 
Ericsson, K. A., \& Kintsch, W. (1995). Long-term working memory. Psychological Review, 102(2), 211-245.

Ericsson, K. A., Krampe, R. T., \& Tesch-Römer, C. (1993). The role of deliberate practice in the acquisition of expert performance. Psychological Review, 100(3), 363-406.

Ericsson, K. A., \& Lehmann, A. C. (1996). Expert and exceptional performance: Evidence and maximal adaptation to task constraints. Annual Review of Psychology, 47(1), 273.

Ericsson, K. A., \& Smith, J. (1991). Prospectus and limits of the empirical study of expertise: An introduction. In K. A. Ericsson, \& J. Smith (Eds.), Toward a general theory of expertise: Prospects and limits (pp. 1-38). Cambridge; New York: Cambridge University Press.

Ertmer, P. A. (1999). Addressing first- and second-order barriers to change: Strategies for technology integration. Educational Technology Research and Development, 47(4), 47-61.

Ertmer, P. A., Conklin, D., Lewandowski, J., Osika, E., Selo, M., \& Wignall, E. (2003). Increasing preservice teachers' capacity for technology integration through the use of electronic models. Teacher Education Quarterly, 30(1), 95-112.

Feltovich, P. J., Johnson, P. E., Moller, J. H., \& Swanson, D. B. (1984). LCS: The role and development of medical knowledge in diagnostic expertise. In W. J. Clancey, \& E. H. Shortliffe (Eds.), Readings in medical artificial intelligence: The first decade. Readings, MA: Addison Wesley.

Hannafin, M. J., Hannafin, K. M., Land, S. M., \& Oliver, K. (1997). Grounded practice and the design of constructivist learning environments. Educational Technology Research and Development, 45(3), 101-117.

Hannafin, M. J., Land, S. M., \& Oliver, K. (1999). Open learning environments: Foundations, methods, and models. In C. M. Reigeluth (Ed.), Instructional-design theories and models: A new paradigm of instructional theory (pp. 115-140). Mahwah, NJ: Lawrence Erlbaum Associates.

Kim, H. (2005). Situated learning with cases: Web-enhanced case-based reasoning in teacher education. Doctoral dissertation, University of Georgia, Athens.

Kim, H., \& Hannafin, M. J. (2005a). Preservice teachers' case-based reasoning (CBR) and situational understanding in the web-enhanced CBR learning environment. Paper presented at the Annual Conference of the American Educational Research Association, Montreal, Canada.

Kim, H., \& Hannafin, M. J. (2005b). Supporting preservice teachers' authentic activity and learning in the web-enhanced case-based reasoning (CBR) learning environment: A case study. Paper presented at the Annual Conference of the Association for Educational Communication and Technology, Orlando, FL, USA.

Kim, H., Hannafin, M. J., \& Kim, M. C. (2005). Online case-based learning: Components, applications, and assessment. Distance Learning, 1(5), 23-31.

Kim, H., Hannafin, M. J., \& Thomas, G. (2004). Helping preservice teachers become experienced: Case-based reasoning (CBR) and grounded design of the web-enhanced learning environment. Paper presented at the Annual Conference of the American Educational Research Association, San Diego, CA.

Kolodner, J. L. (1993). Case-based reasoning. San Mateo, CA: Morgan Kaufmann Publishers.

Kolodner, J. L., Hmelo, C. E., \& Narayanan, N. H. (1996). Problem-based learning meets casebased reasoning. Paper presented at the International Conference on the Learning Sciences, Northwestern University, Evanston, IL.

Kolodner, J. L., \& Guzdial, M. (2000). Theory and practice of case-based learning aids. In D. H. Jonassen, \& S. M. Land (Eds.), Theoretical foundations of learning environments (pp. 215242). Mahwah, NJ: L. Erlbaum Associates.

Kolodner, J. L., Owensby, J. N., \& Guzdial, M. (2004). Case-based learning aids. In D. H. Jonassen (Ed.), Handbook of research on educational communications and technology (pp. 829-861). Mahwah, NJ: L. Erlbaum Associates.

Krueger, K., Boboc, M., Smaldino, S., Cornish, Y., \& Callahan, W. (2004). INTIME impact report: What was INTIME effectiveness and impact on faculty and preservice teachers? Journal of Technology and Teacher Education, 12(2), 185-210.

Lave, J., \& Wenger, E. (1991). Situated learning: Legitimate peripheral participation. Cambridge: Cambridge University Press.

Leinhardt, G., \& Greeno, J. G. (1986). The cognitive skill of teaching. Journal of Educational Psychology, 78(2), 75-95.

Masoner, M. (1988). An audit of the case study method. New York: Praeger. 
Office of Technology Assessment (OTA). (1995). Teachers and technology: Making the connection (OTA-HER-616). Washington, DC: U.S. Government Printing Office.

Owensby, J. N., \& Kolodner, J. L. (2002). Case application suite: Promoting collaborative case application in learning by design classrooms. Paper presented at the International Conference on Computer Support for Collaborative Learning, University of Colorado, Boulder, CO.

Reeves, T. C., Herrington, J., \& Oliver, R. (2005). Design research: A socially responsible approach to instructional technology research in higher education. Journal of Computing in Higher Education, 16(2), 96-116.

Riesbeck, C. K. (1996). Case-based teaching and constructivism: Carpenters and tools. In B. G. Wilson (Ed.), Constructivist learning environments: Case studies in instructional design (pp. 4961). Englewood Cliffs, NJ: Educational Technology Publications.

Russell, M., Bebell, D., O'Dwyer, L., \& O'Connor, K. (2003). Examining teacher technology use: Implications for preservice and inservice teacher preparation. Journal of Teacher Education, 54(4), 297-310.

Schank, R. C. (1993/1994). The design of goal-based scenarios. Journal of the Learning Sciences, 3(4), 305-345.

Schank, R. C. (1999). Dynamic memory revisited (2nd ed.). Cambridge: Cambridge University Press.

Schank, R. C., Berman, T. R., \& Macpherson, K. A. (1999). Learning by doing. In C. M. Reigeluth (Ed.), Instructional-design theories and models: A new paradigm of instructional theory (pp. 161-181). Mahwah, NJ: Lawrence Erlbaum Associates.

Schön, D. A. (1983). The reflective practitioner: How professionals think in action. New York: Basic Books.

Shulman, L. S. (1992). Toward a pedagogy of cases. In J. H. Shulman (Ed.), Case methods in teacher education (pp. 1-30). New York: Teachers College Press.

Shulman, L. S. (1986). Those who understand: Knowledge growth in teaching. Educational Researcher, 15, 4-14.

Spiro, R. J., Feltovich, P. J., Jacobson, M. J., \& Coulson, R. L. (1992). Cognitive flexibility, constructivism, and hypertext: Random access instruction for advanced knowledge acquisition in ill-structured domains. In T. M. Duffy, \& D. H. Jonassen (Eds.), Constructivism and the technology of instruction: A conversation (pp. 57-75). Hillsdale, NJ: Lawrence Erlbaum Associates.

Stevens, A. L., Collins, A., \& Goldin, S. E. (1982). Misconceptions in students' understanding. In D. Sleeman, \& J. S. Brown (Eds.), Intelligent tutoring systems (pp. 13-24). Orlando, FL: Academic Press.

Turns, J. A., Newstetter, W., Allen, J., \& Mistree, F. (1997). The reflective learner: Supporting the writing of learning essays that support the learning of engineering design through experience. Paper presented at the 1997 American Society of Engineering Educators Conference, Milwaukee, WI.

Wang, F., \& Hannafin, M. J. (2005). Design-based research and technology-enhanced learning environments. Educational Technology Research \& Development, 53(4), 5-23.

Windschitl, M., \& Sahl, K. (2002). Tracing teachers' use of technology in a laptop computer school: The interplay of teacher beliefs, social dynamics, and institutional culture. American Educational Research Journal, 39(1), 165-205.

Hyeonjin Kim is Researcher of Policy Planning Office at the Korea Education \& Research Information Service.

Michael J. Hannafin is Professor of Instructional Technology in the Department of Educational Psychology \& Instructional Technology and Director of the Learning \& Performance Support Laboratory at the University of Georgia. 